\title{
Global existence and blow-up to a class of degenerate parabolic equation with time dependent coefficients
}

\author{
Anyin $\mathrm{Xia}^{1,2, a}$, Xianxiang $\mathrm{Pu}^{3, \mathrm{~b}}$, Shan $\mathrm{Li}^{4, \mathrm{c}, \mathrm{d}}$ \\ ${ }^{1}$ Department of Mathematics, Sichuan University, Chengdu, \\ 610065, P.R.China. \\ ${ }^{2}$ School of Science, Xihua University, Chengdu, \\ 610039, P.R.China. \\ ${ }^{3}$ Sichuan Sanhe College of Professional, Luzhou, 646200, P.R.China. \\ ${ }^{4}$ Business School, Sichuan University, Chengdu, 610064, P.R.China. \\ axay004@163.com, 'bzpuxx@163.com, 'lishan@scu.edu.cn \\ ${ }^{\mathrm{d} C}$ Corresponding author
}

Keywords: Degenerate parabolic equation, Global existence, Blow-up.

Abstract. This paper deals with the global existence and blow-up to nonnegative solution of a degenerate parabolic equation with time dependent coefficients under homogeneous Dirichlet boundary conditions. We establish the results on global existence and blow up solution to the system.

\section{Introduction}

In this work, we consider the following degenerate parabolic equation with time dependent coefficients under homogeneous Dirichlet boundary condition

$$
\begin{cases}u_{t}=\Delta u^{m}+f(t) u^{p},(x, t) \in \Omega \times(0, T), \\ u(x, t)=0, & (x, t) \in \partial \Omega \times(0, T), \\ u(x, 0)=u_{0}(x), & x \in \Omega .\end{cases}
$$

Here $\Omega \in R^{N}$ is a bounded domain with smooth boundary $\partial \Omega, \mathrm{m}>1, \mathrm{p}>0$. $\mathrm{f}(\mathrm{t})$ is a positive bounded continuous function with for any $t \geq 0$. The initial value $u_{0}(x)$ is nontrivial nonnegative continuous function and vanishes on $\partial \Omega$.

Global existence and singularity analysis of the solutions to the nonlinear parabolic equation have been investigated in the past decade, please see the famous surveys [1,2].Many physical phenomena have formulated into similar mathematical models(see[2,3,4,5,8,9]).

In [8], Payne and Philippin have considered the linear diffusion case, namely $m=1$.However, the degenerate diffusion makes the present problem more complicated and takes more essential difficulties here.

The purpose in this paper is to investigate some sufficient conditions to the global existence and nonexistence to the solution to the boundary value problem (1). We would like to refer some results on blowup solutions to the degenerate parabolic equations and system in $[2,6,7]$ and references therein.

\section{Global solution for the problem (1)}

In this section, by constructing some global upper solution, we establish the global existence of the solution of problem (1).

Theorem 1. If $p<m$ and $f(t) \leq \bar{k}(\bar{k}:$ const), then every classical solution to the problem (1) is 
global.

Proof: Obviously, if $0<p<m$, then there exists a positive constant $l$ such that $0<m l<1$, $m l-p l>0$.

Now, we construct supersolution which is bounded for any $T>0$. Let $\varphi(x)$ be the solution of the following elliptic problem

$$
\begin{cases}-\Delta \varphi(x)=1, & x \in \Omega, \\ \varphi(x)=0, & x \in \partial \Omega .\end{cases}
$$

Denote $C=\max _{\Omega} \varphi(x)$. Namely, $0 \leq \varphi(x) \leq C$.

We define the function $\bar{u}(x, t)$ as

$$
\bar{u}(x, t)=[K(\varphi(x)+1)]^{l},
$$

where $l$ satisfy $m l<1$ and $K>0$ will be fixed later. Clearly, $\bar{u}(x, t)$ is bounded for any $t>0$. Thus, we have

$$
\begin{aligned}
\bar{u}_{t}-\Delta \bar{u}^{-m} & =-m l K^{m l}\left\{(m l-1)[\varphi(x)+1]^{m l-2}|\nabla \varphi(x)|^{2}+[\varphi(x)+1]^{m l-1} \Delta \varphi(x)\right\} \\
& \geq-m l K^{m l}[\varphi(x)+1]^{m l-1} \Delta \varphi(x)=m l K^{m l}[\varphi(x)+1]^{m l-1} \\
& \geq m l K^{m l}(1+C)^{m l-1},
\end{aligned}
$$

and

$$
f(t) \bar{u}^{p}(x, t) \leq \bar{k}[K(\varphi(x)+1)]^{p l} \leq \bar{k}[K(1+C)]^{p l} .
$$

Denote

$$
K_{1}=\left[\frac{\bar{k}}{m l}(1+C)^{p l-m l+1}\right]^{\frac{1}{m l-p l}} .
$$

As $m l-p l>0$, we can choose $K$ sufficiently large that $K>K_{1}$ and

$$
[K(1+\varphi(x))]^{l} \geq u_{0}(x) .
$$

Now, it follows from (3)-(6) that $\bar{u}(x, t)$ defined by (2) is a positive supersolution of problem (1). Hence $u(x, t) \leq \bar{u}(x, t)$ by comparison principle, which implies $u(x, t)$ exists globally.

\section{Blow-up solution for the problem (1)}

In this section, we focus on the blow-up solution of problem (1).

Theorem 2. If $p \geq m$ and $\underline{k}:=\inf _{\Omega} f(t)>0$, then every classical solution to the problem (1) blows up in finite time.

Proof: Due to the requirement of the comparison principle in problem (1) that we will construct blow-up subsolutions in some subdomain of $\Omega$ in which $u(x, t)>0$. Some ideas borrow from the work [4] by Du.

Let $\psi(x)$ is a nontrivial nonnegative continuous function and vanish on $\partial \Omega$. Without loss of generality, we assume that $0 \in \Omega$ and $\psi(0)>0$.

We will construct a blow-up subsolution to complete the proof.

Set

$$
\underline{u}(x, t)=\frac{1}{(T-t)^{l}} \omega^{\frac{1}{m}}\left(\frac{|x|}{(T-t)^{\mu}}\right),
$$

with 


$$
\omega(r)=\frac{R^{3}}{12}-\frac{R}{4} r^{2}+\frac{1}{6} r^{3}, r=\frac{|x|}{(T-t)^{\mu}}, 0 \leq r \leq R,
$$

where $l, \mu$ and $T>0$ are be determined later.

Clearly, $0 \leq \omega(r) \leq \frac{R^{3}}{12}$ and $\omega(r)$ is nonincreasing since $\omega^{\prime}(r)=\frac{r(r-R)}{2} \leq 0$.

Note that

$$
\operatorname{supp} \underline{u}(\cdot, t)=B\left(0, R(T-t)^{\mu}\right) \subset B\left(0, R T^{\mu}\right) \subset \Omega,
$$

for sufficiently small $T>0$.

Obviously, $\underline{u}(x, t)$ becomes unbounded as $t \rightarrow T$ at the point $x=0$.

Calculating directly, we obtain

$$
\begin{aligned}
\underline{u}_{t}(x, t)-\Delta \underline{u}^{m}(x, t) & =\frac{m l \omega^{\frac{1}{m}}(r)+\mu r \omega^{\prime}(r) \omega^{\frac{1-m}{m}}(r)}{m(T-t)^{l+1}}+\frac{R-2 r}{2(T-t)^{m l+2 \mu}}+\frac{(N-1)(R-r)}{2(T-t)^{m l+\mu}} \\
\leq & \frac{l\left(\frac{R^{3}}{12}\right)^{\frac{1}{m}}}{(T-t)^{l+1}}+\frac{N R-(N+1) r}{2(T-t)^{m l+2 \mu}},
\end{aligned}
$$

notice that $T>0$ is sufficiently small.

Case1: If $0 \leq r \leq \frac{N R}{N+1}$, we have $\omega(r) \geq \frac{(3 N+1) R^{3}}{12(N+1)^{3}}$, then

$$
f(t) \underline{u}^{p}(x, t)=f(t) \frac{1}{(T-t)^{p l}} \omega^{\frac{p}{m}}(r) \geq \frac{\underline{k}}{(T-t)^{p l}}\left(\frac{(3 N+1) R^{3}}{12(N+1)^{3}}\right)^{\frac{p}{m}} .
$$

Hence

$$
\underline{u}_{t}(x, t)-\Delta \underline{u}^{m}(x, t)-f(t) \underline{u}^{p}(x, t) \leq \frac{l\left(\frac{R^{3}}{12}\right)^{\frac{1}{m}}}{(T-t)^{l+1}}-\frac{\underline{k}}{(T-t)^{p l}}\left(\frac{(3 N+1) R^{3}}{12(N+1)^{3}}\right)^{\frac{p}{m}} .
$$

Case2: If $\frac{N R}{N+1}<r \leq R$, then

$$
\underline{u}_{t}(x, t)-\Delta \underline{u}^{m}(x, t)-f(t) \underline{u}^{p}(x, t) \leq \frac{l\left(\frac{R^{3}}{12}\right)^{\frac{1}{m}}}{(T-t)^{l+1}}+\frac{N R-(N+1) r}{2(T-t)^{m l+2 \mu}} .
$$

If $p \geq m>1$, then there exist positive constant $l$, such that $(m-1) l>1$. Thus, we get

for all $\mu>0$.

$$
p l \geq m l>l+1, m l+2 \mu>l+1
$$

Hence, for sufficiently small $\mu>0$ and $T>0$, (8) holds. And (11)-(12) imply that

$$
\underline{u}_{t}(x, t)-\Delta \underline{u}^{m}(x, t)-f(t) \underline{u}^{p}(x, t) \leq 0,
$$

where $(x, t) \in B\left(0, R(T-t)^{\mu}\right) \times(0, T)$.

Since $\psi(x)$ is a nontrivial nonnegative continuous function and $\psi(0)>0$, there exist two positive constants $\rho$ and $\varepsilon$ such that $\psi(x)>\varepsilon$ for all $x \in B(0, \rho)$. Choose $T$ small enough to insure $B\left(0, R(T-t)^{\mu}\right) \subset B(0, \rho)$, hence $\underline{u}(x, t) \leq 0$ on $\partial B\left(0, R(T-t)^{\mu}\right) \times(0, T)$.

From (8), it follows that $\underline{u}(x, 0) \leq \bar{M} \psi(x)$ for sufficient large $\bar{M}$. By comparison principle, we have $\underline{u}(x, t) \leq u(x, t)$ provided that $u_{0}(x) \geq \bar{M} \psi(x)$, which implies that the solution $u(x, t)$ of problem (1) blows up in finite time. 


\section{Remark}

With aid of the differential equality, we can establish lower and upper estimate to the blow up time in our future works.

\section{Acknowledgements}

This work is supported by Project Foundation by Ministry of Education (No. 14YJA630024).

\section{References}

[1] C. Bandle, H. Brunner: J. Comput. Appl. Math., Vol 97 (1998), 3--22.

[2] L. L. Du, C. L. Mu, and M. S. Fan: Dyn. Syst., Vol.20 (2005), 401--412.

[3] L. L. Du: J. Math. Anal. Appl., Vol 324 (2006), 304--320.

[4] L. L. Du: J. Comput. Appl. Math., Vol 202 (2007), 237--247.

[5] M. S. Fan, C. L. Mu and L. L. Du: Appl. Math. Sci., Vol 1 (2007), 13--23.

[6] J. Hernndez, F. Mancebo and J. Vega: Proc. Roy. Soc. Edinburgh Sect., Vol A 137(1) (2007), 41--62.

[7] F. C. Li, C. H. Xie: Appl. Math. Lett., Vol 16 (2003), 185--192.

[8] L. E. Payne, G. A. Philippin: Appl. Math., Vol 3 (2012), 325--330.

[9] L. E. Payne, G. A. Philippin: Proc. Amer. Math. Soc., Vol 141 no. 7 (2013),2309--2318. 